\title{
Methods in Enzymology, Vol. 478, Glycomics
}

\author{
(M. Fukuda, ed., Elsevier, Amsterdam-Boston-Heidelberg-London-New-York-Oxford-Paris, \\ 2010, 611 p., \$145.60)
}

DOI: $10.1134 / \mathrm{S} 0006297911050117$

This and another two volumes of "Methods in Enzymology" (volumes 479 and 480) published by Elsevier Publishing House are a logical continuation of the same series published in 2006 under the editorship of Professor Minoru Fukuda. I fully agree with the Editor that for the last several years glycobiology as an important direction of research has made tremendous progress in immunology, neuroglycobiology, glycomics, signal transduction, and many other disciplines. Explosive progress has been made not only in theoretical glycobiology, but also in new technologies developed specifically for the glycobiology field. Therefore, the reviewed book and other two volumes contain updated and new glycobiology data and methods and, as I do believe, will be a very comprehensive handbook for many specialists in the field.

This book consists of three sections including 27 chapters written by an international group of authors.

The first section considers glycomics revealed by mass spectrometric analysis. Five chapters (1-5) of this section deal with mass spectrometric analysis of sulfated $\mathrm{N}$ - and $\mathrm{O}$-glycans including applying this method for analysis of mutant mice, characterization of glycosaminoglycans, cancer-associated $\mathrm{N}$-glycans, and analysis of in vitro and in vivo enzymatic syntheses of $\mathrm{N}$ and O-glycans.

The second section (chapters 6-14) considers methods of glycomics analysis using carbohydrate-binding proteins. There are characterizations of fucosylated haptoglobin as a novel tumor marker for pancreatic cancer. Several chapters are related to lectins and glycosaminoglycans diagnostic applications, detection of weak-binding sugar activity using membrane-based carbohydrates, fluorescence-based solid-phase assays to study glycanbinding protein interactions with glycoconjugates, multifaceted approaches including neoglycolipid oligosaccha- ride microarrays to ligand discovery for malectin, and imaging mass spectrometry of glycolipids.

The third section of this book (chapters 15-27) describes methods of chemical glycobiology. The separate chapters of this section highlight dynamics and interactions of glycoconjugates probed by stable-isotope-assisted nuclear magnetic resonance spectroscopy, synthetic chemistry for recognition of bacterial and animal glycans, characterization of multivalent ligands for siglecs, and intramolecular glycan-protein interactions in glycoproteins. The specialized chapters of this section include various syntheses of amino-containing oligosaccharides and aminoglycosides, solid phase synthesis of oligosaccharides, and novel synthesis of functional mucin glycopeptides containing both $\mathrm{N}$ - and $\mathrm{O}$-glycans. The last four chapters highlight synthesis of glycopeptides, synthetic approach for gangliosides, metabolic labeling of glycoconjugates with photocrosslinking sugars, and identification of carbohydrate-binding proteins by carbohydrate mimicry peptides.

The book contains author and subject indexes, bibliography for each chapter, and also color photographs placed at the end of the book.

This book is very informative and is novel both in terms of problems discussed and also new methods used for glycobiology. This is also typical for the two subsequent volumes of "Methods in Enzymology" (Vols. 479 and 480), which will be also reviewed in Biochemistry (Moscow).

This book will be very useful for glycobiologists and bioorganic chemists as well as for researchers working in molecular biology and biotechnology. It can also be recommended for university students and their teachers as supplemental material for the above-mentioned fields of science. 